\title{
Nasal Carriage of Staphylococcus in Health Care Workers in Benghazi Hospitals
}

\author{
Nadia. E. Al-Abdli ${ }^{1, *}$, Saleh.H. Baiu ${ }^{2}$ \\ ${ }^{1}$ Department of Laboratory, Eye Hospital, Benghazi, Libya \\ ${ }^{2}$ Department of Botany, Faculty of Science, Benghazi University, Libya \\ *Corresponding author: batul.gr155@gmail.com
}

Received May 07, 2014; Revised June 21, 2014; Accepted July 08, 2014

\begin{abstract}
This study was aimed to determine the frequency of staphylococcal nasal carriage of health care workers (HCWs) and antimicrobial susceptibility profile of the isolates in hospitals of Benghazi, Libya. The study was conducted in the period between April 2013 and August 2013 on HCWs from ten hospitals in Benghazi-Libya. Nasal swabs from anterior nares of HCWs were cultured and identified as Staphylococcus aureus (S. aureus), coagulase - negative staphylococci (CoNS) and methicillin-resistant S. aureus (MRSA) by using standard methods. Antimicrobial susceptibility testing was performed on Muller Hinton Agar using disc diffusion method. Of the 472 HCWs, (47.5\%) were nasal carriers of S. aureus, (21.4\%) for MRSA and (26.1\%) for MSSA, (36.4\%) of CoNS. The highest carriage rate for S. aureus was in Physician (30.6\%) followed by nurses (20.4\%), helpers (22.9\%) and technicians (10.9\%). Almost all of the isolates showed high resistance against penicillin (97.5\%) and ampicillin (98.2\%). The lowest resistance recorded was for augmentin (14.2\%), gentamycin (7.1\%), clindamycin (7.5\%) ciprofloxacin (3.2\%) and vancomycin (2.1\%). Most of isolated strains were susceptible to rifampicin (98.9\%). The emergence of resistant strains of S.aureus should be prevented by controlling the amount of antibiotics used in and out of hospitals. Also, better control measures of nosocomial infection must be established.
\end{abstract}

Keywords: Nasal carriage, health care workers, Staphylococcus aureus, Staphylococcus

Cite This Article: Nadia. E. Al-Abdli, and Saleh.H. Baiu, "Nasal Carriage of Staphylococcus in Health Care Workers in Benghazi Hospitals.” American Journal of Microbiological Research, vol. 2, no. 4 (2014): 110-112. doi: 10.12691/ajmr-2-4-1.

\section{Introduction}

Staphylococcus is a major nosocomial pathogen, both methicillin-sensitive Staphylococcus aureus (MSSA) and methicillin-resistant $S$. aureus are important causes of healthcare-associated infections. Healthcare workers (HCWs) may carry pathogenic hospital strains in their nose and skin, where (HCWs) are vectors of transmission [1]. Accordingly colonized healthcare workers (HCWs) may constitute a reservoir for transmission of resistant organisms like MRSA to patients and other HCWs [2], and may spread these pathogens to the community leading to more dreadful condition [3]. HCWs, who have direct contact with persistently colonized patients, or contaminated objects in the immediate environment around them can contaminate their hands and subsequently transmit the organism to other patients. A subset of these will remain as nasal carrier for a prolonged period of time and may spread the organism to patients by direct contact transmission [4].

The fact that huge portions of healthy population carry $S$. aureus in their nose and body surfaces is responsible for the fast spread of the staphylococcal infections and the situation seems worse in hospitals [5]. So, study of $S$. aureus as nasal carrier is of importance, especially in health care workers to explore the clear picture regarding its existence. Other staphylococci such as coagulase negative Staphylococcus (CoNS) are also pathogenic. Methicillin - resistant CoNS (MRCoNS) have also been found worldwide. Moreover, CoNS may transfer its resistance to MRSA [6,7]. Almost 25\% of the health care workers are stable nasal carriers, and $30 \%$ to $50 \%$ of them also possess the bacteria on their hands [8,9]. Occasionally, health care workers who carry $S$. aureus in their nares are at high risk of infection and can be a potential source of nosocomial pathogens. The aim of this study was to describe the pattern of colonization with Staphylococcus among HCWs of Benghazi Hospitals in Libya as well as the antibiotic susceptibility pattern of these isolates.

\section{Material and Methods}

\subsection{Collection of Samples and Isolation of Bacteria}

This study was performed from April to August 2013 in ten hospitals of Benghazi, Libya. (Psychiatric hospital and Al-Erada sanatorium, Benghazi Medical Center, 7th of October hospital, Benghazi Childrens hospital, AlJoumhouria hospital, Cardiac Center, Nephrology Center, Al-Jala Hospital, Urology and ENT Centers and Eye 
hospital). This study was done on a total of 472 HCWs. The ages of all study population were from 20 year to 65 year.

\subsection{Cultivation and Identification}

Specimens were collected from the anterior nares with sterile dry cotton swabs (SPA Cultiplast, Melano-Italy), dipped in normal saline (0.9\%). All swabs were inoculated on blood agar (BA-HiMedia, India) and subsequently on Mannitol salt agar plates (MSA-HiMedia, India) and were incubated at $37^{\circ} \mathrm{C}$ for $24-48$ hours. Well isolated colonies were initially Gram-stained and then biochemical tests such as catalase, DNase and coagulase tests [10] and API Staph (BioMérieux, France). Characterized according to Bergey's.

\subsection{Methicillin-Resistance Test}

Methicillin resistance was tested using Mueller- Hinton agar with Cefoxitin disc $(30 \mu \mathrm{g})$ by Kirby-Bauer disc diffusion method [11]. Zone diameters were measured and recorded after a $24 \mathrm{~h}$ incubation at $37^{\circ} \mathrm{C}$. A zone size of $>22 \mathrm{~mm}$ was considered sensitive and $<21$ was considered resistant [12].

\subsection{Antibiotic Susceptibility Testing}

Standardized Kirby-Bauer disc diffusion method, was performed on Mueller-Hinton agar. Single isolated colonies were selected and inoculated in Mueller-Hinton broth and placed in incubator for 24 hours at $37^{\circ} \mathrm{C}$. When its turbidity is comparable to 0.5 McFarland turbidity standards, the plates were inoculated with each broth culture and left to dry before the application of antibiotic discs. The plates were inverted and incubated at $35-37^{\circ} \mathrm{C}$ for 18-24 hours. Results were interpreted according to the criteria of CLSI (2012) [12].

\section{Results}

Prevalence of various microorganisms in health care workers a mong the 472 samples, 396 (83.9\%) samples were positive for at least one of the staphylococcal species. Nasal carriage of staphylococci was (83.9\%), comprising of (47.5\%) S. aureus and (36.4\%) CoNS (Figure 1).

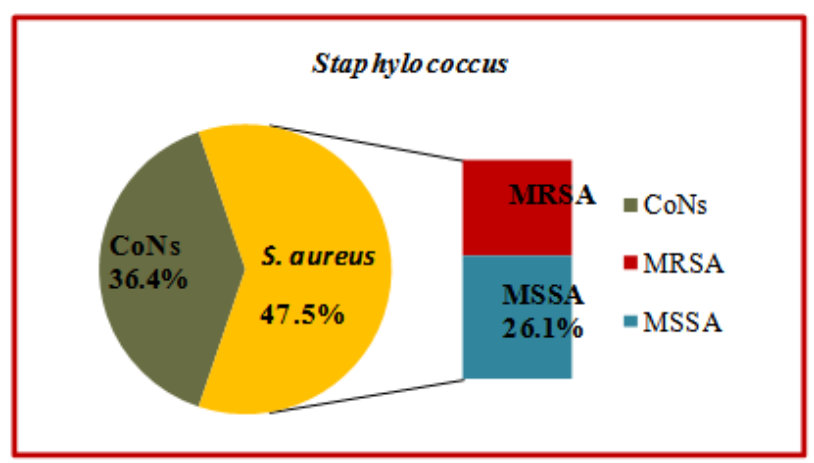

Figure 1. Percentage of Staphylococcus among study population

All study population was screened for MRSA colonization (Table 1). The overall MRSA colonization among (HCWs $n=472$ ) at Benghazi Hospitals was 101 (21.4\%). Of hospital group colonized with MRSA 37
(30.6\%) were Physician, 34 (20.4\%) nurses, helpers 19 (22.9\%), and technicians 11 (10.9\%). This shows that the high carriage rate of MRSA was observed among Physician (30.6\%) compared with other HCWs.

Table 1. Distribution of nasal staphylococci

Table 1. Distribution of nasal staphylococci
\begin{tabular}{|c|c|c|c|c|c|c|c|}
\hline \multirow{3}{*}{ Category } & \multirow{2}{*}{ No } & \multicolumn{6}{|c|}{ Staphylococcus Carriers } \\
\cline { 3 - 8 } & & MRSA & M & No & & No & \% \\
\cline { 3 - 8 } & 121 & 37 & 30.6 & 29 & 24 & 53 & 52 \\
\hline Physician & 167 & 34 & 20.4 & 46 & 27.5 & 60 & 35.9 \\
\hline Nurses & 83 & 19 & 22.9 & 26 & 31.3 & 39 & 47 \\
\hline Helpers & 101 & 11 & 10.9 & 22 & 21.8 & 20 & 20 \\
\hline Technicians & 101 & 21.4 & 123 & 26.1 & 172 & 36.4 \\
\hline HCWs & 472 & 101 &
\end{tabular}

After the identification of $S$. aureus, susceptibility test was done by both Disk diffusion method. A total of 281 strains of $S$. aureus were isolated and tested for their antimicrobial sensitivity patterns. Almost all of the isolates showed high resistance against penicillin where, approximately (97.5\%) of the isolates being resistant to this drug. High rates of resistance to ampicillin (98.2\%), Fusidic acid (61\%), amoxicillin (58.4\%), cefoxitin (43.8\%), oxacillin (42.7\%) and erythromycin (35.9\%) were observed with strains isolated from all population study. The lowest resistance recorded was for augmentin (14.2\%), gentamycin (7.1\%), clindamycin (7.5\%) ciprofloxacin (3.2\%) and vancomycin (2.1\%). On the other hand, most of isolated strains were susceptible to rifampicin (98.9\%). The percentages of antibiotics resistant, susceptible and intermediate in Benghazi hospitals are shown in (Table 2).

Table 2. Antibiotic sensitivity profile of $S$. aureus isolates

Table 2. Antibiotic sensitivity profile of S. aureus isolates
\begin{tabular}{|c|c|c|c|c|c|c|}
\hline \multirow{2}{*}{ Antibiotics } & \multicolumn{2}{|c|}{ Resistant } & \multicolumn{2}{c|}{ Susceptible } & \multicolumn{2}{c|}{ Intermediate } \\
\cline { 2 - 8 } & ${ }^{*}$ No & $\%$ & ${ }^{*}$ No & $\%$ & ${ }^{*}$ No & $\%$ \\
\hline Penicillin & 274 & 97.5 & 7 & 2.5 & - & - \\
\hline Ampicillin & 276 & 98.2 & 5 & 1.8 & - & - \\
\hline Cefoxitin & 123 & 43.8 & 157 & 55.9 & 1 & 0.4 \\
\hline Gentamycin & 20 & 7.1 & 259 & 92.2 & 2 & 0.7 \\
\hline Ciprofloxacin & 9 & 3.2 & 272 & 96.8 & - & - \\
\hline Fusidic acid & 171 & 61 & 110 & 39.1 & - & - \\
\hline Augmentin & 40 & 14.2 & 241 & 85.8 & - & - \\
\hline Erythromycin & 101 & 35.9 & 172 & 61.2 & 8 & 2.8 \\
\hline Clindamycin & 18 & 6.4 & 263 & 93.6 & - & - \\
\hline Cotrimoxazole & 16 & 5.7 & 264 & 94 & 1 & 0.4 \\
\hline Oxacillin & 120 & 42.7 & 151 & 53.7 & 10 & 3.6 \\
\hline Rifampicin & 3 & 1.1 & 278 & 98.9 & - & - \\
\hline Amoxicillin & 164 & 58.4 & 117 & 41.6 & - & - \\
\hline Vancomycin & 6 & 2.1 & 238 & 84.7 & 37 & 13.2 \\
\hline
\end{tabular}

\section{Discussion}

The current study explores Nasal Carriage of Staphylococcus in Health Care Workers in Benghazi Hospitals. In the present study the prevalence of $S$. aureus carriage was $47.5 \%$; out of these $21.4 \%$ were MRSA and CoNS carriage rate was $36.4 \%$ among HCWs, with some differences between hospitals. These rates are higher than those reported in other areas of the world [13,14]. Also Similar results were reported by $[15,16]$. These rates are lower than those reported in other areas of the world $[17,18,19,20]$. All differences between countries and hospitals may be explained by microbiological methods (from sampling technique to culture media), local infection control.

In this study MRSA carriage was particularly high among Physicians, helpers, nurses. These rates are Similar 
than those reported in other areas of the world [16,19,21]. The present study disclosed MRSA isolated from HCWs samples at Benghazi Hospitals were multidrug- resistant (MDR) and highly resistant (100\%) to Beta-lactam antibiotics. These findings are similar to other studies [22,23].

This study showed that MRSA were Vancomycinresistant and Vancomycin intermediately susceptible $S$. aureus. This results agreed with finding of [24,25,26,27].

\section{Conclusion}

The present study indicated a high incidence of nasal carriage of staphylococci among health care workers. It also demonstrated that health personnel are at high risk of infection and can be a potential source of nosocomial pathogens, like $S$. aureus. Therefore, further support is required, recommendations could be made to introduce routine MRSA screening of health care workers as part of a suite of infection control measures and continuous surveillance and improvement of hygiene standards in hospitals should be adopted.

\section{References}

[1] Shenoy, M.S, Bhat, G.K, Kishore, A, Hassan, M.K, Significance of MRSA strains in community-associated skin and soft tissue infections, 2010, 28 (2). 152-154.

[2] Kirecci E, Miraloglu M, A research of nasal methicillin resistant/ sensitive staphylococcus aureus and pharyngeal beta-hemolytic streptococcus carriage in Midwifery students in kahramanaras, Turkey Ethiop J Health Dev 2010, 24 (1): 57-60.

[3] Nakanishi, M, Shrestha, H.G, Rai, S.K, Text Book of Medical Laboratory Technology, 1st Edition. Medical Education Project JICA, TUTH, Kathmandu Nepal. 1996; pp. 359.

[4] Boyce, J.M, Preventing Staphylococcal Infections by Eradicating Nasal Carriage of Staphylococcus aureus. Infect Control Hosp Epidemiol. 1996; 18: 622-7.

[5] Sah, P, Raj Rijal, K,Shakya,B, Raj, B.T,Ghimire,P, Nasal Carriage Rate of Staphylococcus aureus in Hospital Personnel of National Medical College and Teaching Hospital and their Antibiotic Susceptibility Pattern, JHAS, 2013; 3 (1) : 21-23.

[6] Sefani, S and Varaldo, P.E, Epidemiology of methicillin resistant staphylococci in Europe. Clin. Microbiol. Infect, 2003, 9: 11791186.

[7] Hurdle, J.G, O’Neill, A.J, Mody, L, Chopra, I and Bradely, S.F, In vivo transfer of high level mupirocin resistant Staphylococcus aureus associated with failure of mupirocin prophylaxisis. $J$. Antimicrob. Chemother, 2005, 56: 1166-1168.

[8] Luzar, M.A, Coles, G.A, Faller, B, Slingeneyer, A, Dah, G.D, Briat, C, Wone, C, Knefati, Y, Kessler, M and Peluso, M, Staphylococcus aureus nasal carriage and infection in patients on continuous, ambulatory peritoneal dialysis. N. Engl. J Med, 1990, 322: 505-509.

[9] Cespedes, C, Miller, M, Quagliarello, B, Vavagiakis, P, Klein, R.S and Lowy, F.D, Differences between Staphylococcus aureus Isolates from Medical and Nonmedical Hospital Personnel. J. Clin. Microbiol,. (2002), 40: 2594-2597.

[10] Cheesbrough, M, District Laboratory Practice in Tropical Countries. Cambridge university, UK, 2009, 2 (2): (65-67).
[11] Bauer, A. W; Kirby, W. M; Sherris, J. C. and Turck, M, Antibiotic susceptibility testing by a standard single disk method. Am. J. Clin. Pathol,1966; 45: 493-496.

[12] Clinical and Laboratory Standards Institute, Performance Standards for Antimicrobial Susceptibility Testing, Twentysecond Informational Supplement. CLSI document M100-S22. Wayne. PA, 2012.

[13] Bauer, A. W, Kirby, W. M, Sherris, J. C. and Turck, M, Antibiotic susceptibility testing by a standard single disk method. Am. J. Clin. Pathol, 1966, 45: 493-496.

[14] Fadheel, Z.H, Perry, H.E, Henderson, R.A, Comparison of methicillin-resistant Staphylococcus aureus (MRSA) carriage rate in the general population with the health worker population. $N Z J$ Med Lab Science, 2008, 62: 4-6.

[15] Mohammad Bagher, K, Mohammad Kazem, S.Y, Nasal Colonization rate of Staphylococcus aureus strains among Health Care Service Employee's of Teaching University Hospitals in Yazd. Acta Medica Iranica, 2009,47 (4): 315-317.

[16] Zorgani, A, Elahmer,O, Franka,E,Grera, A, Abudher, A, Ghengheah, K.S, Detection of meticillin-resistant Staphylococcus aureus among healthcare workers in Libyan hospitals. Journal of Hospital Infection, 2009, 73: 91-92.

[17] Belgasim, Z,Saadaoui, A, Zorgani, AScreening for methicillinresistant Staphylococcus aureus among health care workers in the African Oncology Institute, Sabrata-Libya. American Journal of Infection Control, 2010, 38 (6): 498-499.

[18] Fadeyi, A, Bolaji, B.O, Oyedepo, O.F, Methicillin Resistant Staphylococcus aureus Carriage amongst Healthcare Workers of the Critical Care Units in a Nigerian Hospital. Am J Infect Dis, 2010, 6 (1): 18-23.

[19] Truong, H; Shah, S.S; Ludmir, J; Twananana, E.O; Bafana, M; Wood S.M; Moffat, H; Steenhoff, A.P. (2011). Staphylococcus aureus skin and soft tissue infections at a tertiary hospital in Botswana. S Afr Med J 101 (6): 413-416.

[20] Ahmed, M.O, Elramalli, A.K, Amri, S.G, Abuzweda, A.R, and Abouzeed, Y.M, Isolation and screening of methicillin-resistant Staphylococcus aureus from health care workers in Libyan hospitals. Eastern Mediterranean Health Journal La Revue de Santé de la Mediterranean Orientale, EMHJ, 2012, 18 (1): 37-42.

[21] Shibabaw, A, Abebe, T, Mihret, A, Nasal carriage rate of methicillin resistant Staphylococcus aureus among Dessie Referral Hospital Health Care Workers; Dessie, Northeast Ethiopia. Antimicrobial Resistance and Infection Control, 2013, 2: 25.

[22] Al brich, W.C, and Harbarth, S, Healthcare workers: Source, vector or victim of MRSA?. Lancet Infect. Dis, 2008, 8: 289-301.

[23] YU, C; Karr,T; Turner,D; Towns, V and Sinha, J, Rapid Detection of Beta-lactamase Production in Penicillin Sensitive Staphylococci by the Phoenix ${ }^{\mathrm{TM}}$ Automated ID/AST System. As presented at the 9th European Congress of Clinical Microbiology and Infectious Diseases (ECCMID), March 1999, Berlin, Germany.

[24] Elgadi, S.A, Carriage rate and antibiotic sensitivity of Staphylococcus aureus in \& outside Children's hospital-Benghazi Libya. An M.Sc, Gar younis University, Faculty of medicine, 2000.

[25] Al alem, A.M, Antibiotic resistant S.aureus infection studies in hospitals. A thesis, Middle East Technical University, 2008.

[26] Al Geobory, H. A, Comparative study between Methicillin resistant Staphylococcus aureus (MRSA) and Methicillin sensitive Staphylococcus aureus (MSSA), and detect the antimicrobial effects of some plant extracts on them. M.Sc. thesis. College of Science. Baghdad University, 2011.

[27] Al Zaidi, J.R, Al Sulami, A.A, Comparison of chromogenic agar medium and diffusion disk test for detection of hospital acquired methicillin resistant Staphylococcus aureus (HA-MRSA) from patients and hospital environment in Nasiriyah city, Iraq. Academic Journal, 2013, 7 (19): 1888-1895. 